\title{
STEFAN BANACH 1892-1945
}

News came recently that Stefan Banach died in Europe shortly after the end of the war. The great interest aroused in this country by his work is well known. In fact, in one of Banach's main fields of work, the theory of linear spaces of infinitely many dimensions, the American school has developed and continues to contribute very important results. It was a rather amazing coincidence of scientific intuition which focused the work of many mathematicians, Polish and American, on this same field, a field which grew so richly in the period between the two world wars. Actually, it was E. H. Moore who was the precursor of the doctrine of applying abstract algebraic methods to many concrete problems of analysis. It is not necessary to describe the achievements of Banach and his students which center around his monograph on the theory of linear operations.

Banach's work brought out for the first time in the general case the success of the methods of geometrical and algebraic approach to problems in linear analysis-far beyond the more formal discoveries of Volterra, Hadamard, and their successors. His results embraced more general spaces than the work of such mathematicians as Hilbert, Schmidt, Riesz, von Neumann, Stone, and others. ${ }^{1}$ Many mathematicians, especially the younger ones in the United States, took up this idea of geometrical and algebraic study of linear function spaces, and the work is still going on vigorously and producing rather important results. Also, it is not necessary to stress the possibilities of the application of similar methods of approach to problems of nonlinear analysis, at present so numerous and important for immediate applications. Banach had a number of results (unfortunately unpublished) on the theory of polynomials and analytical operators in a class of suitably defined infinitely-dimensional spaces.

He had in preparation a sequel to his volume on linear operators dealing with spaces on which one could study nonlinear transformations. Various approaches to the study of "nonlinear analysis" have of course been made before by mathematicians. In the opinion of the writer the proper definition of a natural class of spaces on which these more general operators should be studied has not yet been found. Very

1 The initial general formulation was published, almost simultaneously with that of Banach, by Norbert Wiener who, however, changed his field of interest very shortly afterward. 
often in the history of mathematical theories one of the factors deciding the success of a new discipline is the proper amount of generality for the class of objects under discussion. It should contain a large class of examples already existing in mathematics and not be overburdened by too many arbitrary constructions.

Banach's contributions to fields of mathematics grouped around the theory of functions of real variables, set theory, and general groups are equally important and include some of the most elegant and final results in these fields. To give only a few examples, the specialist will remember his theorems on functions of bounded variation, the theorems on differentiability of functions, and his paper on the length of curves and the area of surfaces. His work of the early twenties was characterized by extreme elegance and perspicacity of proofs. One remembers the originality of his proof of Vitali's decomposition theorem and the beautiful set-theoretical investigations on one-to-one transformations of abstract sets. His proof of the theorem stating that a set which is locally of first category is of first category in the large, even for the non-separable case, is an example of his ingenuity. ${ }^{2}$ Of course, most mathematicians know Banach's results on measure in Euclidean spaces and in abstract sets (containing the results obtained jointly with Tarski on the paradoxical decomposition of two spheres of different radii into an equal number of pairwise congruent sets). Banach's theories of generalized limits.for sequences of numbers and of functions, a joint work with Mazur, find a growing number of applications. He wrote also two interesting papers on general metric groups.

When we survey his work, the role played by the set-theoretical and topological methods in more classical mathematical disciplines becomes obvious. For instance, the very important work of Schauder (a student of Banach who was killed during the war) in which he establishes existence theorems in the theory of differential equations uses the method of general function spaces. As other examples of the success of these methods of analysis one has only to point out Banach's work in the theory of general orthogonal series or general integrals.

Among Banach's writings, in addition to his well known monograph on linear operations, are a university textbook on differential and integral calculus in two volumes and a university text on mechanics - the latter, in the opinion of the writer, a masterful presentation, extremely worth translating into English.

${ }^{2}$ This theorem was still further generalized in an interesting paper by Deane Montgomery. 
Banach was born in 1892. His family was poor and he had very little conventional schooling at first. Thus he was mostly self-educated when he went to the Polytechnical Institute in Lwów. It is said that it was Steinhaus who accidentally discovered Banach's talent by overhearing a mathematical conversation between Banach and another student on a park bench in Cracow. Banach and Steinhaus were to become later the closest collaborators and the senior members of the Lwow school of mathematics. Banach was a student at the Polytechnical Institute between 1910 and 1914. He obtained his Ph.D. in 1920, and was instructor at the Polytechnical Institute between 1920 and 1922, became a docent and an "extraordinary professor" at the Lwów University in 1922, and a professor in 1929. He was a member of the Polish Academy of Sciences and won several prizes including the prize of the Academy and a scientific prize of the City of Lwów. The writer has no precise knowledge of his life and work from the outbreak of the war to his premature death in the fall of 1945 .

Banach worked in periods of great intensity separated by stretches of apparent inactivity. During the latter, however, his mind kept working on the selection of statements that would best serve as focal theorems in the next field of study. He liked constant mathematical discussion with friends and students. The writer remembers a mathematical session with Mazur and Banach lasting seventeen hours without interruption except for meals. In general both the Lwow and Warsaw mathematical schools were fond of and successful in collaboration. A large proportion of the papers published were written by more than one author. It was Banach and, in Warsaw, Sierpinski who were mainly responsible for this development.

Much of the mathematical work was carried on in a way not usual in American mathematical centers. The mathematicians in Lwow met not only in their classrooms and offices but spent long hours every day in two coffee houses which served as informal meeting places. They discussed problems over coffee or beer, and marble table tops and napkins took the place of blackboards. It was hard to outlast or outdrink Banach during these sessions. He was always there. The weekly (Saturday night!) meetings of the mathematical society, where papers were presented, provided the more formal discussions.

Banach's faculty for proposing problems illuminating entire sections of mathematical disciplines was very great. These, properly formulated, can of ten play the role of experimenta crucis in a physical theory. The constant challenge of these questions, proposed by mathematicians of the group over which Banach presided, stimulated the gradual progress of the theories, now so well known, in functional 
analysis and real variables. He took up eagerly the challenge of any important question connected with the domain in which he was working at the time, and he could spend months on it.

Banach's publications reflect only a part of his mathematical powers. The diversity of his interests in mathematics surpasses by far that shown in his papers. His personal influence on other mathematicians in Lwów, and in Poland, was in many cases decisive. It stands out as one of the main traits of this remarkable period of twenty years when so much mathematical work was accomplished and so many high hopes were raised - all to be interrupted by World War II.

All this is now a rather distant memory. Of the many outstanding mathematicians who were Banach's students, Schauder, Auerbach, and Kaczmarz are dead. All the others have left Lwów. But some members who survived the war are trying to reconstitute the group in the new surroundings of Breslau to which Lwów's university has been transferred.

S. ULAM 